Rev. Latinoam. Psicopat. Fund., São Paulo, v. 14, n. 3, p. 530-541, setembro 2011

\title{
A síndrome de Charles Bonnet e o problema dos transtornos de percepção visual nos idosos
}

German E. Berrios

Peter Brook

A literatura, clássica ou recente, sobre os transtornos de percepção nos idosos é revisada. A utilidade do conceito de Sindrome de Charles Bonnet, um epônimo originalmente proposto para descrever as alucinações visuais nos idosos na ausência de prejuízos cognitivos e de oftalmopatia periférica é contestada, principalmente devido à síndrome ter sido progressivamente ampliada. Descrevemos três casos representativos de pacientes idosos que desenvolveram diferentes tipos de transtornos de percepção visual. Propomos a condução de estudos sistemáticos da frequência de tais transtornos nos idosos e de quais outras patologias, particularmente cognitivas e visuais que possam estar associadas a eles.

Palavras-chave: Síndrome de Charles Bonnet, alucinações visuais história, alucinações - fisiopatologia, percepção visual

* Publicação original: The Charles Bonnet Syndrome and the problem of visual perceptual disorders in the elderly. Age and Ageing, v. 11, n. 1, p. 17-23, 1982. Tradução de Lazslo A. Ávila, revisão técnica de Ana Maria G. R. Oda. 


\section{Introdução}

A expectativa clínica de que transtornos de percepção visual sejam mais comuns entre os idosos (Ey, 1973) não recebeu confirmação estatística. Isso não é de todo surpreendente. A pesquisa sobre a incidência de ilusões e alucinações visuais em todos os grupos etários é obstaculizada por dificuldades definicionais e metodológicas e pela natureza fluida e indeterminada das próprias experiências. No caso dos idosos, isso se compõe de falhas de memória, da qualidade variada e frequentemente bizarra de alguns desses transtornos e da natural relutância do sujeito em admitir esse tipo de sintoma, para não serem considerados doentes mentais (Morsier, 1967; White, 1980).

Tanto a literatura clássica quanto a recente é de pouca ajuda no tópico das alucinações visuais em idosos (Baillarger, 1846; Brierre de Boismont, 1859; Parish, 1897), sendo considerado que a maioria dessas experiências ocorreriam no contexto de estados tóxicos e confusionais (Lipowski, 1980). De fato, as alucinações visuais durante os estados confusionais agudos (Robinson, 1956; Simon \& Cahan, 1963) e o delirium tremens (Gross et al., 1971) são menos comuns nesse grupo etário. Isso tem sido explicado como resultante de demência e, portanto, da pobreza intelectual sobre a qual os estados confusionais agudos podem estar sobrepostos (Lipowski, 1980). Uma situação similar se observa nos estados paranoides, parafrenia e esquizofrenia de início tardio que são menos comumente acompanhadas por alucinações visuais do que nos grupos etários mais jovens. Mesmo quando a psicose paranoide se relaciona com a presença de catarata, as alucinações visuais estão claramente ausentes (Cooper \& Porter, 1976).

Além dos transtornos orgânicos e das psicoses funcionais, as alucinações visuais em idosos podem ocorrer em situações de privação sensorial (por exemplo, cegueira após cirurgia nos olhos) e ainda mais interessante, elas podem ocorrer sem causa aparente e acompanhadas de pleno insight. Um caso desse tipo, descrito pelo suíço Bonnet, no século XVIII, foi citado ao longo do século seguinte para sustentar a ideia de que as alucinações poderiam ocorrer nos indivíduos saudáveis (Brierre de Boismont, 1859) (veja abaixo). 
Este artigo revisa alguns dos problemas relativos ao estudo das alucinações visuais nos idosos e analisa o estatuto clínico da chamada Síndrome de Charles Bonnet. Três casos são descritos (veja o Apêndice).

\section{Alucinações visuais (e fenômenos semelhantes) nos idosos}

Ey (1973) declarou, em seu verbete sobre "alucinações na senescência": "após algum tempo, todos os clínicos percebem a tendência dos idosos em alucinar ... no entanto, a maioria dos profissionais ainda confunde os dois tipos de alucinações visuais". Estes são "aquelas que emergem fora da consciência e são rejeitadas pelo sujeito como experiências falsas e aquelas que se apoiam na desorganização da consciência e são aceitas como autênticas..." De acordo com Ey, a tendência a alucinar é devida à "idade produzir uma deterioração funcional tanto do sistema nervoso central quanto do periférico, favorecendo a liberação dos fenômenos alucinatórios, principalmente eidola". Desdobrando essa ideia (Sutter, 1962; Kanowski, 1978), diversas hipóteses podem ser sugeridas. Em primeiro lugar, a idade pode acarretar uma desintegração dos centros superiores (a la Jackson), e a "liberação" de fenômenos subcorticais (Cogan, 1973). Em segundo lugar, um estado de "desaferentação" pode ser engendrado pela visão ou audição falhas, enfraquecendo assim os controles externos sobre a percepção. Isso, por sua vez, pode liberar mecanismos perceptuais próprios para "completar" estímulos incompletos, ou mesmo criar novos (Ajuriaguerra \& Garrone, 1965; Fischer, 1969; Cordeiro, 1973). Em terceiro lugar, uma irritação cortical pode produzir imagens visuais como na epilepsia do lobo temporal ou na estimulação cortical (Baldwin, 1967; British Medical Journal, 1977). Em quarto lugar, o aumento nas alucinações visuais nos idosos pode ser mais aparente do que real, isso é, pode ser devido não a qualquer "fator etário" como tal, mas à incidência aumentada de estados tóxicos e confusionais (Lipowski, 1980), psicoses funcionais, ou uma combinação desses fatores. Em quinto lugar, algumas alucinações visuais dos idosos podem estar submetidas a controle genético, seja indiretamente pela produção de uma psicose subjacente ou diretamente ao produzir uma propensão a desenvolver transtornos perceptivos isolados (Bergener et al., 1972).

Evidências empíricas que apoiam quaisquer dessas hipóteses são limitadas. Por exemplo, estudos sobre a privação sensorial desde a década de 1950 não foram capazes de demonstrar que os transtornos visuais eliciados fossem alucinações visuais "reais" (Zuckerman, 1970). Os estudos de estimulação cortical de Penfield requerem modificações. O número de experiências alucinatórias eliciadas não é de modo algum grande; o mesmo evento visual se repete raramente 
sob estimulação do mesmo local, a memória para a alucinação é pequena e a alucinação é acompanhada por completa consciência e pouca ou nenhuma resposta emocional (Horowitz \& Adams, 1970). A "arteriosclerose cerebral" não pode ser considerada uma explicação suficiente para a presença das alucinações visuais; a distinção entre as alucinações arterioscleróticas dos "pequenas veias" (Giacomo, 1952), ou dos "grandes veias" (Coiffu, 1958) deve ser revista. Além disso, não há evidências de que uma redução global na perfusão cerebral tenha lugar como resultado apenas da arteriosclerose (Hachinski et al., 1974). Mais aceitável é a ideia de que as alucinações visuais ocorrem como um fenômeno irritativo no contexto de estados de multi-infarto, envolvendo o lobo occipital (Vallet, 1967). O fato de que elas possam ocorrer no campo hemianóptico dá apoio a essa opinião (Lance, 1976). A mesma explicação pode ser relevante para a assim chamada "síndrome da alucinação óptica” de Reimer (1970) e ser o caso nas alucinações visuais formadas na arterite craniana (Hart, 1967). Alucinações visuais também ocorrem nas demências senis do tipo Alzheimer, mas são muito menos comuns na doença de Pick (Ey, 1973); não está esclarecido se isso é um artefato estatístico resultante da incidência muito menor dessa última condição. Alucinações visuais associadas com catarata (Brunerie \& Coche, 1936; Bartlett, 1951), acuidade visual diminuída e cegueira parcial (White, 1980) são os concomitantes clínicos dos transtornos de percepção relacionados à privação sensorial; não se sabe se esses ocorrem mais frequentemente entre idosos. Essa forma de alucinose visual não responde aos neurolépticos (Post, 1965).

Alucinações visuais acompanhadas de insight e que ocorrem na ausência aparente de confusão, doença neurológica, privação sensorial ou transtornos oftalmológicos têm sido descritas como Síndrome de Charles Bonnet (Morsier, 1967). A existência desse estado (como descrito por Morsier) foi contestada por Ey (1973) e Hecaen e Albert (1978), que afirmaram que ela ocorre sempre na presença de acuidade visual reduzida. Essa síndrome é teoricamente relevante no sentido de que seria o único exemplo de alucinações visuais que ocorreriam na ausência de patologia orgânica ou psiquiátrica e acompanhada de insight.

\section{A Síndrome de Charles Bonnet}

O filósofo suíço Charles Bonnet (1720-1793) em seu Essai Analytique sur les Facultés de l'Âme (1769) relatou as experiências bizarras de seu avô, Charles Lullin, um magistrado de 89 anos de idade, de Genebra, a quem Bonnet descreveu como "muito saudável, sensível e sem dificuldades de memória de qualquer tipo". Lullin teve um surto de alucinações visuais que duraram três meses em 1758,

Rev. Latinoam. Psicopat. Fund., São Paulo, v. 14, n. 3, p. 530-541, setembro 2011 
durante o qual ele via "sem qualquer estímulo externo, a imagem de homens, mulheres, pássaros, prédios que mudavam de forma, tamanho e lugar, mas que ele nunca aceitava como reais. O senhor em questão se submeteu, em idade avançada, a operações de catarata em ambos os olhos". Embora não seja claro no texto, pareceria que as operações teriam se realizado antes do episódio alucinatório. Bonnet concluiu que essas alucinações "tiveram lugar naquela parte do cérebro que contém o órgão da visão".

No século XX, Flournoy (1902) localizou um manuscrito de Lullin contendo uma autodescrição de suas experiências, que não corresponde com a narrativa de Bonnet. Lullin declarou que tinha essas visões apenas quando de pé ou sentado, mas nunca quando deitado, e que as visões eram mais claras no seu campo visual esquerdo, desaparecendo quando virava seus olhos para a direita. Mais tarde em sua vida, o próprio Bonnet teve experiências semelhantes e viu, de acordo com seu biógrafo Levêque de Pouilly (1794) "muitos objetos fantásticos que ele reconheceu como ilusórios". A esse respeito é sabido que Bonnet sofreu de uma marcada redução na acuidade visual cedo na vida, em um grau que o forçou a abandonar o uso de seu microscópio (Claparade, 1909).

A descrição de Bonnet tornou-se, durante o século XIX, o paradigma da existência de alucinações visuais nos indivíduos saudáveis (Ey, 1973). Naville (1873) descreveu um caso semelhante e comentou que as alucinações visuais podem coexistir com a razão. Ele também referiu as alucinações visuais experimentadas por Cabanis, que "via claramente pessoas andando em um caminho distante". Naville ofereceu seis critérios para reconhecer essas alucinações que são compatíveis com a razão: 1) elas ocorrem com consciência clara e não enganam o sujeito; 2) elas ocorrem combinadas com percepções normais; 3) elas são exclusivamente visuais; 4) elas não são acompanhadas de quaisquer sensações bizarras; 5) elas aparecem e desaparecem sem qualquer causa óbvia, e 6) elas são divertidas, em vez de aborrecidas para o sujeito. Esses fenômenos foram, ao longo do século XIX, distinguidos das assim chamadas "pseudoalucinações" de Hagen e Kadinski (Ey, 1973). Por exemplo, Fournoy (1923) relatou o caso de um homem de 89 anos, sem déficit intelectual, que sofrera uma operação de catarata, e que via, mesmo de olhos fechados, "senhoras sedutoras, elegantemente vestidas, saindo de uma nuvem (em seu quarto) e então desaparecendo pela janela".

A literatura do começo do século XX contém diversos relatos anedóticos de sujeitos idosos, sofrendo de patologias oculares e experienciando alucinações visuais com consciência clara, com insight e sem nenhum prejuízo intelectual óbvio. Descrições inadequadas e ausência de corroboração post-mortem e psicométricas não permitem nenhuma conclusão clínica sólida. Weinberger e Grant (1940) estudaram os aspectos conceituais e clínicos das alucinações visuais em relação à patologia do córtex visual. Concluíram que as alucinações vi- 
suais não tinham valor de localização, na medida em que outros fenômenos similares podiam resultar de lesões em qualquer nível do aparato visual; a complexidade e tipo de imagens dependiam muito mais de fatores psicológicos e constitucionais do que da "organização cortical". Infelizmente sua amostra de 16 casos (variando entre 10 e 59 anos) não auxilia no exemplo dos idosos.

Morsier propôs o epônimo "Síndrome de Charles Bonnet" em 1938. Posteriormente ele coletou e analisou 18 casos relatados na literatura (com idade média de 81 anos, 11 homens e 7 mulheres) e lutou para demonstrar que "não havia correlação entre o início das alucinações visuais e a patologia dos olhos"; em cinco casos (do total de 15) as alucinações haviam se desenvolvido enquanto os sujeitos tinham visão normal (Morsier, 1967). Então ele definiu a síndrome como: presença de alucinações visuais em idosos, sem evidência de prejuízo cognitivo e não relacionadas etiologicamente com oftalmopatias periféricas. Sugeriu que a causa seria encontrada no próprio cérebro.

Desde a descrição de Morsier, uma visão mais ampla da síndrome se desenvolveu, de acordo com a qual ela se refere a qualquer estado de alucinações visuais nos idosos, independente de sintomatologia acompanhante. Por exemplo, estados de demência (Burgmeister et al., 1964) (Casos 1, 2, 3, Apêndice), estados alucinatórios posteriores a cirurgias nos olhos (Ajuriaguerra \& Garrone, 1965) e acuidade visual diminuída (Hecaen \& Albert, 1978), todos se qualificariam a esse título. Parece que há pouca utilidade em usar um epônimo para descrever todos os estados alucinatórios visuais em idosos, já que é muito mais esclarecedor descrever esses fenômenos em detalhes, especificando o tipo e a severidade da patologia ocular envolvida (por exemplo, White, 1980).

Impressiona a frequencia com que parentes e pessoal de enfermagem relatam como idosos demenciados parecem ter alucinações visuais ou outras distorções de percepção visual, mas sem turvação de consciência. Alguns parecem ver fotografias ou imagens de televisão como se fossem reais (Caso 1), outros mostram tanto experiências ilusórias baseadas em percepções reais quanto alucinações de fato (Caso 2), enquanto outros parecem ter pura alucinose (Caso 3). O que agora é necessário. e o que nos propomos a fazer é conduzir um estudo sistemático de quão frequentemente distúrbios de percepção visual ocorrem nos idosos, sua natureza e fatores associados, tais como a presença de demência, acuidade visual e se a catarata está ou não presente. 


\section{Apêndice}

\section{Caso 1. WFA, data de nascimento, 16/3/1907}

Início súbito das alucinações visuais, provavelmente como um estado confusional agudo, na metade de 1978. Recuperado desse estado agudo, mas demência progressiva se desenvolveu desde então, e a alucinose visual ou erros perceptivos persistiram. Ele vê pessoas no jornal como reais e se comporta como se elas assim o fossem, por exemplo, dando pedaços de comida na boca das fotografias ou então oferecendo drinques a elas. Em uma ocasião, foi encontrado indo ao centro para comprar uma garrafa de uísque para Elisabeth Taylor. Frequentemente vê garotas de topless na página 3 do jornal The Sun como reais, falando com elas, perguntando a elas como estão, quais são seus nomes, e então informando a elas o que ele pretende, finalmente declarando seu amor a elas. Ele lhes dá biscoitos, drinques e cigarros, colocando-os sobre suas bocas, e se no meio de uma conversação com uma dessas garotas ele tem que ir ao banheiro, na volta pergunta: "Para onde foi a garota?". Ele também interpreta erroneamente o que vê em fotografias, por exemplo, ele pode ver um laço no pescoço de uma garota como se fosse uma faca e pedir que ela seja levada ao setor de emergências do Hospital Addenbrookes.

Fisicamente, diabético mal controlado por hipoglicemiantes orais, agora substituídos por insulina. Teve catarata precocemente, mas sua visão está prejudicada severamente, a pressão arterial é 130/95. Nenhum sinal óbvio no sistema nervoso central, sorologia negativa, a CAT scan (Tomografia Axial Computadorizada) mostra atrofia cerebral difusa. Diagnóstico clínico: demência senil, do tipo Alzheimer.

\section{Caso 2. LM, data de nascimento, 7/7/1905}

Início de alucinose visual bastante rápido, mas apenas por pequenos períodos e então por mais longos até que suas alucinações visuais persistiram pelo dia inteiro. Mais ou menos na mesma época, começo de falhas de memória, por volta do início de 1978.

As alucinações tomaram duas formas. A primeira era ver pessoas ou objetos à distância. De acordo com sua esposa essas não eram tanto distorções visuais como objetos reais formando a base para a experiência alucinatória, mas muito alterados, por exemplo, se houvesse uma rosa vermelha no jardim, então a pessoa que ele relatava estar vendo estaria vestindo roupas vermelhas, enquanto dois postes à distância seriam vistos como um trator. As outras alucinações visuais eram sempre de pessoas com faces feias que ele pensava serem amigos de sua 
mulher, a quem podia ver perto da casa, e ele teria forte objeção à sua presença e tentaria afastá-los, e em uma ocasião tentou enforcar a um deles. Essas alucinações não eram formadas sobre a base de qualquer pista visual. Inicialmente, sua esposa conseguia persuadi-lo a sair da experiência, mas apenas temporariamente. Próximo ao final de seu período em casa, ele ficava muito bravo se sua realidade era contestada. Mostrou alguma deterioração na visão distante nos últimos anos, mas é capaz de ler e a visão proximal é aparentemente boa.

\section{Caso 3. LAK, data de nascimento, 7/7/1897}

Início insidioso da demência em junho de 1978, quando foi descrita como "vaga e confusa" e repetindo constantemente as mesmas perguntas. Vista inicialmente em janeiro de 1980, quando a fala era desconexa e discursiva com prejuízos desiguais de memória, sem disfasia mas com as funções do lobo parietal parcialmente prejudicadas. Diagnóstico: demência multi-infarto. História passada de acidentes cerebrovasculares em novembro de 1978, deixando-a com reflexos exagerados no lado direito. Catarata periférica no olho direito, mas com fundo de olho normal.

Início de alucinações visuais no começo de maio de 1980, seguidas pouco depois por acidente vascular cerebral transitório; alucinações visuais persistiram continuamente desde maio de 1980, quando vista pela última vez em dezembro de 1980 e ainda presentes no relato de assistentes sociais em março de 1981. Algumas alucinações persistentes, especialmente a de um bebê pequeno. Aponta pessoas sentadas em cadeira para os visitantes e se torna enraivecida se eles não conseguem percebê-los. No entanto, a paciente se refere às aparições como "fantasmas".

\section{Referências}

Ajuriaguerra, J. de; Garrone, G. Désafferentiation partielle et psychopathologie. In: Désafferentiation expérimentale et clínique (Symposium Bel Air II). Geneva: Georg, 1965. p. 91-157.

Baillarger, M. J. Des hallucinations. Mem. Acad. R. Med., n. 122, p. 273-475, 1846.

BALDwin, T. Visual hallucination. In: Handbook of Clinical Neurology. Vol. 3. Ed.Vinken \& Bruyn. Amsterdam: North-Holland, 1967.

BARTLETT, J.E.A. Hallucinations in an old man with cataracts. Brain, n. 74, p. 363, 1951.

BERGENER, M. et al. Klinische und morphologische Untersuchungen über eine familiäre Altershalluzinose. Nervenarzt, n. 43, p.18-33, 1972. 
Bonnet, Ch. Essai Analytique sur les Facultés de l'Âme. 2. ed. Vol. 11, p. 176-177. Copenhagen and Geneva, 1769.

Brierre de Boismont, A. Hullucinations. London: Henry Renshaw, 1859.

British Medical Journal. Leading Article: Localisation of visual hallucinations. $\mathrm{Br}$. Med. J. 2, p. 147-148, 1977.

Brunerie, A.; Coche, R. Sur trois cas d'hallucinations visuelles chez des cataractés. Ann. Med. Psychol., n. 94, p. 166-171, 1936.

Burgmeister, J.J. et al. Les hallucinations visuelles des ophthalmopathies.

Neuropsychologie, n. 3, p. 9-38, 1964.

Claparade, E. La Psychologie Animale de Charles Bonnet (Memoire publié à l'occasion du jubilé de l'université, 1559-1909), p. 21. Geneva: Georg, 1909.

Cogan, D.G. Visual hallucinations as release phenomena. Albrecht v. Graefes Arch. Klin. Exp. Ophthal, n. 188, p. 139-150, 1973.

CoIfru, B. Artériosclérose cérebrale. Thesis. Paris, 1958 (Quoted by Ey 1973).

CoOper, A. F.; Porter, R. Visual acuity and ocular pathology in the paranoid and affective psychoses of later life. J. Psychosom. Res., n. 20, p. 107-114, 1976.

Cordeiro, J. C. États délirants du troisième age. L'encéphale, n. 62, p. 20-55, 1973.

Ey, H. Traité des hallucinations. 2 vols. Paris: Masson, 1973.

Fischer, R. The perception-hallucination continuum. Dis. Nerv. Syst., n. 30, p. 161-171, 1969.

Flournoy, Th. Le cas de Charles Bonnet. Hallucinations visuelles chez un vieillard opéré de la cataracte. Arch. Psychol. (Geneva), n. 1, p. 1-23, 1902.

FlouRnoy, H. Hallucinations liliputiennes atypiques chez un vieillard opéré de la cataracte. L'encéphale, n. 18, p. 566-579, 1923.

Giacomo (1952). (Quoted by Ey 1973).

Gross, M.M. et al. Hallucinations and clouding of sensorium in alcohol withdrawal. $Q$. J. Stud. Alcohol., n. 32, p. 1061-1069, 1971.

HAChinsKi, V. C. et al. Multi-infact dementia. A cause of mental deterioration in the elderly. Lancet ii, p. 207-209, 1974.

HART, C. T. Formed visual hallucinations: A symptom of crania1 arteritis. Br. Med. J., n. 2, p. 643-644, 1967.

Hecaen, H.; Albert, M. L. Human Neuropsychology. New York: John Wiley, 1978.

Horowitz, M.J.; Adams, J.E. (1920) Hallucinations on brain stimulation: Evidence for revision of the Penfield Hypothesis. In: Origin and Mechanisms of Hallucinations. Ed.: W. Keup. p. 13-22. New York: Plenum Press, 1970. 
Kanowski, S. Classification of psychopathological symdromes in old age. In: Brain Failure in Old Age. Ed: F. Hoffmeister \& C. Müller. Berlin: Springer-Verlag, 1978.

LANCE, J. W. Simple formed hallucinations confirmed to the area of a specific visual field defect. Brain, n. 99, p. 719, 1976.

LeVÊQue de PoullLy, J.S. Eloge de Charles Bonnet. Lausanne: Henbach, 1794. p. 120-121.

Lipowski, Z.J. Delirium. Illinois: Charles C. Thomas, 1980.

Morsier, G. DE. Les hallucinations. Rev. Oto-neuro-ophthalm., n. 16, p. 244-352, 1938. . Le syndrome de Charles Bonnet. Hallucinations visuelles des vieillards sans déficience mentale. Ann. Med. Psychol., n. 125, p. 677-702, 1967.

NAVILle, E. (1873) (Quoted by Morsier 1967).

PARISH, E. Hallucinations and Illusion. London: Walter Scott, 1897.

Post, F. The Clinical Psychiatry of Late Life. Oxford: Pergamon, 1965.

ReImer, F. Das Syndrom der optischen Halluzinose. Stuttgart: Thieme Verlag, 1970.

Robinson, G.W. The toxic delirious reactions of old age. In: Mental Disorder in Latter Life. Ed. O. J. Kaplan. Stanford, Ca.: Stanford University Press, 1956.

Simon, A.; Cahan, R.R. Acute brain syndrome in geriatric patients. Psychiatr. Res. Rep., n. 16, p. 8-21, 1963.

SutTER, J.M. L'apport de la neurologie à la psychopathologie des hallucinations. Evol. Psychiatr., n. 27, p. 501-535, 1962.

VaLLET, R. (1967) Thesis, Paris (Quoted by Ey 1973).

WeIMBERGER, L.M.; Grant, F.C. Visual hallucinations and their neuro-optical correlates. Arch. Ophthal. (Chicago), n. 23, p. 166-199, 1940.

White, N.J. Complex visual hallucinations in partial blindness due to eye disease. $B r$. J. Psychiatr., n. 136, p. 284-286, 1980.

ZuCKerman, M. Reported sensations and hallucinations in sensory deprivation. In: Origin and Mechanisms of Hallucinations. Ed.: W. Keup. New York: Plenum Press, 1970. p. 133-148.

\section{Resumos}

(The Charles Bonnet syndrome and the problem of visual perceptual disorders in the elderly)

A review is presented here of the classical and recent literature on perception disorders in the elderly. The author contests the usefulness of the concept of the Charles 
Bonnet syndrome, a category first proposed to describe visual hallucinations in elderly persons in the absence of cognitive damage or peripheral ophthalmopathy. This objection is brought up mainly because the concept has been progressively broadened. We describe here three representative cases of elderly patients who developed different types of disorders in visual perception. Systematic studies are now needed on the frequency of such disorders in the elderly and on the question as to what other pathologies, especially of the cognitive and visual type, may be associated with them.

Key words: Charles Bonnet syndrome, visual hallucinations - history, and hallucinations - physiopathology, visual perception

(Le syndrome de Charles Bonnet et le problème des troubles de perception visuelle chez les personnes âgées)

Cet article présente une révision de la littérature classique et récente sur les troubles de la perception des personnes âgées. L'utilité du concept du syndrome de Charles Bonnet, un éponyme proposé à l'origine pour décrire des hallucinations visuelles chez les personnes âgées sans troubles cognitifs, ni d'ophtalmopathie périphérique, est contestée, surtout à partir du fait que ce syndrome a été progressivement élargi. Nous décrivons trois cas représentatifs de patients âgés qui ont développé de différents types de troubles de perception visuelle. Nous suggérons des études systématiques sur la fréquence de ces troubles chez les personnes âgées et sur les autres maladies, en particulier cognitives et visuelles, qui peuvent y être associées. Mots clés: Syndrome de Charles Bonnet, hallucinations visuelles - histoire, hallucinations - physiopathologie, perception visuelle

(El síndrome de Charles Bonnet y el problema de los trastornos de percepción visual en personas viejas)

Se revisa la literatura clásica o reciente sobre los trastornos de percepción en viejos. Se cuestiona la utilidad del concepto de Síndrome de Charles Bonnet, un epónimo, originalmente propuesto para describir las alucinaciones visuales en viejos en ausencia de prejuicios cognitivos y de oftalmopatologia periférica, principalmente por causa de una ampliación progresiva de ese síndrome. Se describen tres casos representativos de pacientes de edad que desarrollaron diferentes tipos de trastornos de la percepción visual. Proponemos la conducción de estudios sistemáticos de la frecuencia de esos trastornos en viejos y de cualquier otras patologias, particularmente cognitivas y visuales que puedan estar asociadas a ese síndrome.

Palabras clave: Síndrome de Charles Bonnet, alucinaciones visuales - historia, alucinaciones - fisiopatologia, percepción visual 
Citação/Citation: BerRios, G.E.; BrooK, P. A síndrome de Charles Bonnet e o problema dos transtornos de percepção visual nos idosos. Revista Latinoamericana de Psicopatologia Fundamental, São Paulo, v. 14, n. 3, p. 530-541, set.2011.

Editor do artigo/Editor: Prof. Dr. Manoel Tosta Berlinck

Recebido/Received: 15.7.2010 / 7.15.2010 Aceito/Accepted: 25.9.2010 / 9.25.2010

Copyright: () 2009 Associação Universitária de Pesquisa em Psicopatologia Fundamental/ University Association for Research in Fundamental Psychopathology. Este é um artigo de livre acesso, que permite uso irrestrito, distribuição e reprodução em qualquer meio, desde que o autor e a fonte sejam citados/This is an open-access article, which permits unrestricted use, distribution, and reproduction in any medium, provided the original author and source are credited.

Financiamento/Funding: Os autores declaram não ter sido financiados ou apoiados/The authors have no support or funding to report.

Conflito de interesses/Conflict of interest: Os autores declaram que não há conflito de interesses/The authors declare that has no conflict of interest.

\section{German E. BERRIOS}

Médico e filósofo pela Universidad Nacional de San Marcos, Lima, Peru; Psiquiatra; Neurologista; Psicólogo; Filósofo; Historiador e Filósofo da ciência (Oxford University, England); Professor de Neuropsiquiatria e de Epistemologia da Psiquiatria (University of Cambridge, England), desde 1976; Neuropsiquiatra e Chefe do Departamento de Neuropsiquiatria do Hospital Addenbrooke, University of Cambridge, por 32 anos; Coordenador do Comitê de Ética em Pesquisa com Humanos na mesma universidade, por 20 anos; Editor Responsável de History of Psychiatry; Autor de 14 livros, incluindo The History of Mental Symptoms, Descriptive Psychopathology since 19th Century (Prêmio Nacional BMA, 1997), A History of Clinical Psychiatry (com Roy Porter), e Delirio (com F. Fuentenebro) e mais de 400 artigos e capítulos de livros; Membro do Royal College of Psychiatrists; da Associação Britânica de Psicologia e da Academia Britânica de Ciências Médicas; Membro Vitalício do Robinson College, Cambridge; doutor Honoris-Causa da Universidade de Heidelberg (Alemanha), da Universidade Nacional Mayor de San Marcos (Peru) e da Universidad Autónoma de Barcelona (Espanha); Grão Oficial da Ordem del Sol (Condecoração do Governo Peruano, 2007); prêmio Ramon y Cajal 2008 concedido pela Asociación Internacional de Neuropsiquiatria.

University of Cambridge

Box 189, Hills Road

Cambridge,

UK CB2 2QQ

e-mail: geb11@cam.ac.uk

\section{Peter Brook}

Professor associado da Cambridge University; Consultor Psicogeriátrico do Fulbourn Hospital. Cambridge CB1 5EF 